\title{
La paz en los discursos del presidente Juan Manuel Santos*
}

\section{Peace in the speech of President Juan Manuel Santos}

\author{
Jairo Alonso Páez Lancheros** \\ Edilberto Fagua Jiménez ${ }^{* * *}$
}

Fecha de recepción: 5/08/2017

Fecha de aceptación: 2/10/2017

\section{Resumen}

El presente artículo de reflexión tiene por objeto evidenciar la relación que existe entre la propuesta del presidente Santos para la terminación del conflicto armado, la construcción de una paz estable y duradera, contenida en sus dos discursos de posesión y la

* Artículo de reflexión que analiza de manera crítica desde la perspectiva del Análisis Crítico del discurso los discursos del presidente Juan Manuel Santos.

** Jairo Alfonso Páez Lancheros, Profesional Universitario en Comunicación Social de la Universidad Jorge Tadeo Lozano Sede Bogotá; Licenciado en Música de la Universidad Pedagógica Nacional Sede Bogotá y Candidato a Magíster en Derechos Humanos de la Universidad Pedagógica y Tecnológica de Colombia UPTC, seccional Chiquinquirá. Actualmente se desempeña como Directivo Docente Coordinador de la Institución Educativa Instituto Técnico Industrial «Julio Flórez» de Chiquinquirá. Correo electrónico: jairopaez61@gmail.com

*** Edilberto Fagua Jiménez, Licenciado en Ciencias Sociales y Económicas de la Universidad Pedagógica y Tecnológica de Colombia UPTC, seccional Chiquinquirá; especialista en Educación Personalizada de la Universidad Católica de Manizales, y Candidato a Magister en Derechos Humanos de la Universidad Pedagógica y Tecnológica de Colombia UPTC seccional Chiquinquirá. Actualmente se desempeña como Directivo Docente -Rector de la Institución Educativa Hato Viejo de San Miguel de Sema. Correo electrónico: jairopaez61@gmail.com 
continuidad de la Doctrina de la Seguridad Nacional. Para el logro de las metas planteadas, se ha tomado como metodología el Análisis Crítico del Discurso (ACD), de Teun Van Dijk.

\section{Palabras clave}

Análisis Crítico de Discurso, Paz y Doctrina de la Seguridad Nacional.

\section{Abstract}

This article reflects on the relationship between the proposal of President Santos for the end of the armed conflict, the construction of a stable and lasting peace contained in his two speeches of possession, and the continuity of the Doctrine of the National Security. In order to achieve the stated goals, the Critical Analysis of the Discourse of the Teun Van Dijk has been take as methodology.

\section{Key Words}

Critical Analysis of the Discourse, Peace and Doctrine of National Security.

\section{Introducción}

El propósito de este artículo de reflexión es hacer un análisis crítico de los discursos del presidente Juan Manual Santos, emitidos al tomar posesión del cargo como mandatario de los colombianos en su primer periodo, el 7 agosto de 2010, y al ser reelegido para un segundo periodo de gobierno, el 7 de agosto de 2014, para identificar las intencionalidades proyectadas en sus alocuciones en torno a su propuesta de paz para el país. 
Al seleccionar, como objeto de estudio, las dos intervenciones del presidente Santos, se pretende identificar en cada disertación los elementos que permitan evidenciar la relación entre la propuesta de terminación del conflicto armado, la construcción de una paz estable y duradera, toda vez que la paz es el eje transversal para la vigencia y garantía de los demás derechos humanos; sin embargo, al mantener las estrategias de la Doctrina de la Seguridad Nacional, contenidas en el Plan Colombia, se conserva implícita la guerra como salida al conflicto armado.

\section{El análisis Crítico de Discurso ACD.}

El discurso es definido por Teun Van Dijk como un acontecimiento comunicativo, es decir, un fenómeno práctico, político y social que no se reduce únicamente a la emisión de oraciones gramaticalmente correctas, sino a la interacción social al desarrollarse dentro de un contexto específico, de ahí que los usuarios del lenguaje adecúen su comportamiento de acuerdo a diversas categorías sociales. (Van Dijk, 2003)

\section{El Análisis Crítico del Discurso ACD es considerado como}

Un campo académico heterogéneo e interdisciplinar que empieza a gestarse en forma paralela en diferentes disciplinas desde mediados de los años 60, que adquiere una progresiva institucionalización (revistas, congresos, cursos de formación, núcleos de investigación, manuales, etc.) a partir de los años 80, y cuyo principal interés es el estudio empírico de los usos del lenguaje, hablado o escrito, en sus contextos (macro y/o micro) sociales específicos (Tusón, 1999).

De otra parte, el ACD se ha convertido en el campo donde convergen los aportes de diferentes disciplinas ...es una expresión de creciente interés de la ciencias sociales y humanas por discutir e investigar el lugar del lenguaje en la vida social, destacando su carácter de acción y práctica social, así como sus efectos productivos y constitutivos a nivel de los saberes, las interacciones y las subjetividades 
que definen la especificidad de un particular ordenamiento socio histórico (Iñiguez, 2003).

Como afirma Ruth Wodak el objetivo central es estudiar las relaciones de dominación, discriminación, poder y control, tal como se manifiestan a través del lenguaje (Wodak, 2009). En otras palabras, el ACD se propone investigar de forma crítica la desigualdad social tal como viene expresada, señalada, constituida, legitimada, etc., por los usos del lenguaje (es decir en el discurso); o como lo señala Van Dijk el foco del ACD es "el estudio crítico de la reproducción discursiva de la dominación en la sociedad" (Stecher, 2010, p.). Entonces, el investigador que aborda el ACD adquiere una herramienta que le permite develar el trasfondo del discurso y evidenciar las intencionalidades que hay tras las frases, palabras, recursos literarios empleados por el enunciador.

Van Dijk afirma que «el ACD debería ser esencialmente diverso y multidisciplinar. El ACD puede realizarse en, o combinarse con, cualquier enfoque y subdisciplina de las humanidades y las ciencias sociales» (Van Dijk, 2003). Esta característica es valiosa para el desarrollo de investigaciones desde diferentes campos de la ciencia, pues permite transitar por caminos diversos para llegar a la comprensión de las pretensiones, intereses, objetivos y demás propósitos del emisor, sin que haya lugar a adoptar un método definido; sin embargo, permite al analista tener autonomía y dominio en la selección del objeto, teorías, métodos y principios para realizar su análisis; lo importante es el aporte en la compresión de los fenómenos sociales, culturales, económicos, etc., abordados.

\section{La paz como derecho humano o estrategia política.}

La referencia a la paz como un derecho humano, nos remite a la Constitución Japonesa del 3 de noviembre de 1946, que en su preámbulo reconoce que 
Todos los pueblos del mundo tienen derecho a vivir en paz (...) aseveración que es ratificada en el artículo 9 del mismo cuerpo normativo, por el cual Japón toma la determinación de... Renunciar para siempre a la guerra como derecho soberano de la nación, y al uso de la amenaza o la fuerza como un medio de resolución de disputas internacionales (Escobar, 2011).

En el ámbito de las Naciones Unidas, la Carta de San Francisco de 1945, considerada como la declaración internacional fundadora de las Naciones Unidas, expresa como propósito del organismo, en su artículo 1, parágrafo 1. «Mantener la paz y la seguridad internacional y con tal fin, tomar medidas colectivas eficaces para prevenir y eliminar amenazas a la paz y para suprimir actos de agresión u otros quebrantamientos de la paz» (Naciones, uniandes.edu.co, 2006).

La Carta de las Naciones Unidas, en su artículo 2, parágrafo 4, establece que

Los miembros de la organización, en sus relaciones internacionales se abstendrán de recurrir a la amenaza o al uso de la fuerza contra integridad territorial o la independencia política de cualquier Estado o en cualquier otra forma incompatible con los propósitos de las Naciones Unidas (Naciones, uniandes.edu.co, 2006).

En la Declaración de las Naciones Unidad sobre el Derecho de los Pueblos a la Paz, proclama solemnemente «los pueblos de nuestro planeta tienen el derecho sagrado a la paz» (Naciones, uniandes. edu.co, 2006). Sin embargo, como lo señala Escobar, esta declaración «estableció como obligación para los gobiernos el hacer sus mejores esfuerzos para colaborar en la implementación del derecho de los pueblos a la paz, a través de la adopción de medidas apropiadas en los niveles nacional e internacional» (Escobar, 2011).

Si bien, la legislación internacional no le confiere a la paz el reconocimiento como derecho humano, en el marco de la Conferencia General de la Unesco, celebrada en Paris entre el 21 de octubre y el 12 noviembre de 1997, se hace un intento valioso a través de la Propuesta de Declaración de la Paz como Derecho Humano (Unesco), 
proposición presentada en el marco de las deliberaciones de la Conferencia y que no fue aprobada por los delegatarios.

Sin embargo, en el plano internacional existen diferentes reconocimientos de la paz como derecho, tales como el Derecho de los Estados, en la carta de las Naciones Unidas, con la prohibición de la guerra; el derecho de los pueblos, en el artículo 23 de la Carta Africana sobre Derechos Humanos y de los Pueblos de 1981, y en la ya señalada Declaración sobre el Derecho de los Pueblos a la Paz de 1984; en la Resolución 5/XXXII de la Comisión de Derechos Humanos de 1976 (Comisión Interamecricana, 1976), y en la Resolución 33/73 de la Asamblea General, sobre Preparación de las Sociedades para la Vida en Paz, de 1978. (Naciones, 1978).

En el ámbito nacional, la Constitución Política de Colombia es considerada como una «Constitución para la Paz». Desde el preámbulo se establece que,

En ejercicio de su poder soberano, representado por sus delegatarios a la Asamblea Nacional Constituyente, invocando la protección de Dios, y con el fin de fortalecer la unidad de la Nación y asegurar a sus integrantes la vida, la convivencia, el trabajo, la justicia, la igualdad, el conocimiento, la libertad y la paz, dentro de un marco jurídico, democrático y participativo que garantice un orden político, económico y social justo, y comprometido a impulsar la integración de la comunidad latinoamericana, decreta, sanciona y promulga la siguiente Constitución (Constitución Política de Colombia, 2016).

En el desarrollo del articulado constitucional, la paz como derecho y deber, está contenida en el artículo 22 cuando afirma: "la paz es un derecho y un deber de obligatorio cumplimiento" (Constitución Política de Colombia, 2016). Este artículo hace de la carta política un pacto para el logro de la paz, en el cual se permite la vigencia de los Derechos Humanos, la soberanía y la República Democrática.

El Artículo 67 de la Constitución, al definir y determinar los fines de la educación, establece que «La educación formará al colombiano en el respeto a los derechos humanos, a la paz y a la democracia; 
y en la práctica del trabajo y la recreación, para el mejoramiento cultural, científico, tecnológico y para la protección del ambiente» (Constitución Política de Colombia, 2016). Para dar cumplimiento a este precepto constitucional, se establecieron áreas como la Ciencias Sociales, que incluyen como objeto de estudio la Constitución Política.

$\mathrm{Al}$ definir el régimen político colombiano como una república presidencial, la Carta Constitucional en el Artículo 188 puntualiza el significado de la figura del primer mandatario «El presidente de la República simboliza la unidad nacional y al jurar el cumplimiento de la Constitución y de las leyes, se obliga a garantizar los derechos y libertades de todos los colombianos». (Constitución Política de Colombia, 2016), lo cual implica que sus obligaciones en ejercicio del cargo, se traduzcan en imperativos categóricos frente a la garantía de los derechos.

El carácter imperativo de la norma se refuerza en el artículo 95 al establecer como deber de la persona y el ciudadano: «Propender al logro y mantenimiento de la paz» (Constitución Política de Colombia, 2016), en este sentido, Norberto Bobbio desde el campo jurídico, resalta la importancia de la pretensión de la Constitución colombiana al señalar que

En el ámbito de un orden jurídico pueden perseguirse otros fines, paz con libertad, paz con justicia, paz con bienestar, pero la paz es la condición necesaria para el logro de todos los demás fines, y, por lo tanto, se convierte en la razón misma de la existencia del derecho (Sánchez, 2016).

De otra parte, es pertinente citar pretensiones investigativas para ahondar en el concepto de la paz; para tal fin la propuesta de Lina Escobar brinda una definición, que para el caso colombiano, abre caminos de discusión y acercamiento para el esclarecimiento de la paz como derecho; la investigadora plantea que: «La Paz con justicia significa poner fin a la ordalía, hacer plenas las libertades, hoy arrinconadas, y establecer la vigencia plena de los derechos hu- 
manos y no de la razón de Estado, que es el de la fuerza represiva» (Escobar, 2011).

Según Ricardo Sánchez, Frank Przetacznik presenta una definición que muestra la conexidad de la paz con el respeto y garantía de los derechos humanos:

La paz puede ser definida como existencia de tranquilidad interna dentro de un Estado, basado en el reconocimiento, garantía y estricta observación de los derechos humanos de todos los individuos que habiten en dicho Estado y las relaciones normales con otros Estados, basadas en el reconocimiento y observación de los derechos e intereses jurídicos de cada uno (Przetacznik, (año), citado por Sánchez, 2016).

La mencionada definición tiene en cuenta cuatro aspectos fundamentales: 1.) los derechos humanos básicos 2.); cualquier clase de opresión interna o externa de los individuos o grupos, sea política, económica, ideológica o religiosa, debe estar estricta prohibida por cada Estado 3.); todos los pueblos y las naciones deben garantizar e implementar estrictamente su derecho a la autodeterminación, incluyendo el derecho de secesión 4.) la ocupación, subyugación, o dominación sobre otros pueblos, naciones o Estados por poderes externos, deben ser abolidas (Sánchez, 2016).

Como quiera que la anterior definición parte del respeto a los derechos humanos, la conclusión de Przetacznik resulta decisiva en la pretensión de un concepto a cerca de la paz como derecho

La base filosófica del derecho a la paz emana del derecho humano fundamental a la vida... El derecho a la vida significa que todo ser humano tiene el derecho a no ser privado de su vida. El derecho a la vida presupone que una persona esté viva y tenga así la posibilidad de realizar otros derechos humanos, como el derecho a la paz (Przetacznik, (año), citado por Sánchez, 2016).

El mismo autor señala el deber del Estado en relación con la preservación y respeto del derecho a la paz en el marco de una confrontación bélica, y destaca su conexión directa con el derecho a la vida. 
El deber del Estado de respetar el derecho a la vida de los individuos también incluye la prohibición de iniciar una guerra agresiva contra otro Estado, porque en tal guerra, el derecho a la vida de todas las personas que son asesinadas en la guerra es vulnerado (Sánchez, 2016).

De otra parte, resulta de gran importancia para el propósito conceptual de la paz como derecho humano, hacer un rápido recorrido a lo estipulado por la Corte Constitucional colombiana en su esfuerzo por darle carácter normativo a la paz, lo expresado en la Sentencia C- 695/2002 (Corte Constitucional, 2002); debe entenderse como un ideal, que el sistema jurídico ordena buscar.

La Corte Constitucional ha entendido la paz como "el reinado del derecho y la existencia de cauces institucionales adecuados para resolver los conflictos”, ya que la función del régimen constitucional no es suprimir el conflicto - inmanente a la vida en sociedad - sino regularlos, para que sea fuente de riqueza y se desenvuelva de manera pacífica y democrática. (Colombia C. C., Corte Constitucional, 1994). De otra parte, en la sentencia T- 008 de 1992, la CCC se asume el derecho a la paz como presupuesto de goce de los demás derechos consagrados en la Constitución (Colombia C. C., Corte Constitucional, 1992).

\section{La Guerra, el opuesto a la paz}

Cuando se habla de paz, de manera implícita se hace referencia a la guerra, por lo cual es necesario considerar algunos conceptos sobre este tema en particular. La Corte Constitucional en Sentencia C- 876 de 2002, desde la perspectiva de los deberes del Estado y los ciudadanos en materia impositiva señala:

Se entiende la paz como lo contrario a la guerra... No significa lo anterior, que la paz sea solo lo opuesto a la guerra, significa también cooperación interestatal, y entre los individuos de una nación, aprecio mutuo y conciencia del valor de la seguridad (Colombia C. C., Corte Constitucional, 2002). 
Para el teórico Karl Von Clausewitz «la guerra es una forma de relación entre los humanos que surge con la intención de doblegar o someter al otro o incapacitarlo mediante el desarme» (Medrano B, 2012) y en este caso, la paz se convierte en la salida a la confrontación, llegándose a través del sometimiento del adversario e incluso, mediante la eliminación física de este, sin que esto pueda significar la armonía y el fin del conflicto que dio origen a la contienda bélica.

Si bien, en la guerra se pone en riesgo el derecho a la vida, también es cierto que existen normas que pretenden humanizar el enfrentamiento, regulando a los contendientes como lo hace el Derecho Internacional Humanitario y como lo señala Verri, citado por Medrano (Medrano B, 2012), crea entre los bandos reglamentaciones o formalizaciones para hacer de la guerra, algo no tan malo, $y$ en algunas circunstancias hacerla ver como necesaria para un colectivo. Entonces, la guerra adquiere algunos condicionamientos que hacen que la violación flagrante de los derechos humanos, tenga una condena para quienes incurran en estas violaciones. Además, que, si la justicia local no condena a los victimarios, lo puedan hacer los tribunales internacionales.

\section{Discurso de posesión, agosto 7 de 2010}

Juan Manuel Santos, a lo largo de la campaña por la presidencia de la República en el año 2010, enfatiza en dar continuidad a la política de su antecesor, Álvaro Uribe Vélez. En materia de seguridad, considera que están sentadas las bases firmes para lo que será su programa de gobierno, «Prosperidad Democrática», el cual requiere de la «Unidad Nacional» para consolidar la Colombia que todos soñamos.

$\mathrm{Al}$ asumir el cargo de presidente, Santos esboza una propuesta de paz en cada alocución. Inicia con el anuncio... iLe llegó la hora a Colombia! seguido de frases, «Es el mensaje de la vida, de la 
armonía, y de la unidad dentro de la diversidad...Es el mensaje de la Colombia pacífica y sabía que queremos dejar a nuestros hijos» (Santos J. M., 2010).

Las afirmaciones del mandatario denotan su intencionalidad y propósito de conseguir la paz, y recuerda que, transcurridos doscientos años del grito de independencia, el sueño de los próceres no se ha cumplido, por lo cual «sentirían desilusión, porque doscientos años después no hemos alcanzado la justicia social ni consolidado la paz, soportes de la verdadera libertad, esa libertad por la que ellos vivieron y murieron» (Santos J. M., 2010).

Con la frase: «la puerta del diálogo no está cerrada con llave», el presidente alude a la posibilidad del dialogo y la vez deja enunciadas sus aspiraciones de

sembrar las bases de una verdadera reconciliación entre los colombianos. De un desarme real de los espíritus, construido sobre cimientos perdurables que no alimenten falsas esperanzas, que no permitan más engaños y que no conduzcan a nuevas frustraciones en un país que, desde lo más profundo de su alma ensangrentada, lo que más desea es la paz (Santos J. M., 2010).

Aunque, el logro de la paz es un anhelo de los colombianos, no se puede desconocer que desde la misma implementación del Plan Colombia se ha trazado

Una estrategia de paz que se apunte a unos acuerdos de paz negociados con la guerrilla con base en la integridad territorial, la democracia y los derechos humanos, que, además deban fortalecer el estado de derecho y la lucha contra el narcotráfico (Rodríguez V, 2005).

$\mathrm{Al}$ referirse a las condiciones para el posible diálogo, recoge elementos que están en el sentir de los colombianos: un desarme real, el deseo de paz perdurable y la no repetición de fracasos; una vez más el presidente Santos recoge el sentir del pueblo colombiano «desde lo más profundo de su alma ensangrentada, lo que más desea es la paz» (Santos J. M., 2010). 
De otra parte, hace un llamado a la guerrilla, a quien le reitera su disposición de dialogar, sin dejar de considerarla como al margen de la ley

A los grupos armados ilegales que invocan razones políticas y hoy hablan otra vez de diálogo y negociación, les digo que mi gobierno estará abierto a cualquier conversación que busque la erradicación de la violencia, y la construcción de una sociedad más próspera, equitativa y justa (Santos J. M., 2010).

Ante el propósito de lograr una paz al interior del país, el mandatario lo hace extensivo al contexto regional... «Queremos vivir en paz con todos nuestros vecinos» y coloca como condición el respeto mutuo «Los respetaremos para que nos respeten» (Santos J. M., 2010).

Por otro lado, las promesas de campaña se trasladan al acto de posesión «presidiré un Gobierno que será de Unidad Nacional y que buscará la prosperidad social para todos los colombianos» (Santos J. M., 2010). La prosperidad prometida coincide con la primera estrategia del Plan Colombia en lo referente a la economía y generación de empleo. "Una estrategia económica para generar empleo, que fortalezca la capacidad del estado para recaudar impuestos, y que ofrezca una fuerza económica viable para contrarrestar el narcotráfico". (Rodríguez V, 2005).

El Presidente plantea la unidad como «Un acuerdo en torno a la necesidad de tener una democracia vigorosa; una economía estable y próspera; una patria justa en lo económico y lo social; una Nación segura y en paz».8 El acuerdo propuesto es el reflejo del Plan Colombia o «Plan para la Paz, la Prosperidad y el Fortalecimiento del Estado» (Rodriguez V, 2005), en lo referente a las diez estrategias que apuntan a conservar el modelo económico actual.

El compromiso de campaña de trabajar para disminuir la pobreza, lo compara con la forma de combatir al terrorismo, lo cual 
corresponde a la continuidad de la política de seguridad que su antecesor mantuvo durante sus dos periodos de gobierno "Trabajaremos para disminuir la pobreza con la misma intensidad y con el mismo compromiso con que combatimos -y seguiremos combatiendo- el terrorismo y otros enemigos de Colombia” (Santos J. M., 2010).

La política de seguridad la extiende al ámbito urbano, y la relaciona con el cierre de brechas sociales y de oportunidades para la población, asumiendo la seguridad como tranquilidad, ausencia de miedo y zozobra

Vamos a meterle el hombro a nuestras ciudades, para mejorar su seguridad y su movilidad, y para cerrar las brechas sociales y de oportunidades entre sus habitantes... Pondremos en marcha una estrategia integral de seguridad ciudadana para que los centros urbanos sean espacios sociales, de convivencia, donde los niños, las mujeres, los ancianos, puedan jugar, caminar y vivir tranquilos, isin temor ni zozobra! (Santos J. M., 2010).

Esa misma política de seguridad la extiende al sector rural, para proteger a un campesino transformado en empresario exitoso como el que está plasmado en la figura emblemática, «Vamos a defender al campesino colombiano, vamos a convertirlo en empresario, a apoyarlo con tecnología y créditos, para hacer de cada campesino un próspero Juan Valdez». (Santos J. M., 2010). Nuevamente el Plan Colombia se evidencia, esta vez para denotar la estrategia desarrollo alternativo, que fomente esquemas agropecuarios y otras actividades económicas rentables para los campesinos y sus familias (Rodriguez $\mathrm{V}, 2005)$. Señala al narcotráfico, el terrorismo y la violencia, como fenómenos causantes del despojo de tierras que ha afectado a una buena parte de la población y les advierte: «iEso lo vamos a reversar!» (Santos J. M., 2010).

La relación terrorismo y narcotráfico, es recurrente en el discurso presidencial; define los buenos colombianos, que son mayoría, y los malos minoría, atribuyéndoles la responsabilidad de impedir la 
prosperidad; por lo tanto, la salida es defender a los buenos y combatir a los malos.

En medio de la voluntad y el tesón de más de 45 millones de buenos colombianos, subsiste, sin embargo, una ínfima minoría que persiste en el terrorismo y el narcotráfico, en obstruir nuestro camino hacia la prosperidad...A todas las organizaciones legales las defenderemos y a las ilegales las seguiremos combatiendo sin tregua ni cuartel (Santos J. M., 2010).

El combate al narcotráfico y la guerrilla es otros de los aspectos del Plan Colombia

Una estrategia antinarcóticos, en asocio con los demás países involucrados en algunos o todos los eslabones de la cadena: la producción, distribución, comercialización, consumo, lavado de activos, de precursores y otros insumos, y el tráfico que alimenta la violencia hacia la guerrilla y otras organizaciones armadas (Rodriguez V, 2005).

El presidente Santos destaca los avances en la seguridad y la urgencia de convertirla en prioridad, hasta el punto de llamar a las Fuerzas Armadas para que continúen con la guerra «Con la consolidación de la seguridad democrática hemos avanzado en esta dirección como nunca antes, pero falta camino por recorrer» (Santos J. M., 2010).

Sin embargo, frente la posibilidad de entablar un dialogo con los alzados en armas, afirma «la puerta del diálogo no estaba cerrada con llave» y la supedita a cinco premisas inalterables: «la renuncia a las armas, al secuestro, al narcotráfico, a la extorsión, a la intimidación». Refuerza sus condicionamientos al declarar que «No es la exigencia caprichosa de un gobernante de turno. iEs el clamor de una Nación!» (Santos J. M., 2010).

Al indicar otras condiciones para el diálogo, advierte su intención de continuar con la guerra

Pero mientras no liberen a los secuestrados, mientras sigan cometiendo actos terroristas, mientras no devuelvan a los niños reclutados a la fuerza, mientras sigan minando y contaminando los campos colombianos, seguiremos en- 
frentando a todos los violentos, sin excepción, con todo lo que esté a nuestro alcance... Es posible tener una Colombia en paz, una Colombia sin guerrilla, iy lo vamos a demostrar! Por la razón o por la fuerza (Santos J. M., 2010).

\section{Discurso de posesión, agosto 7 de 2014}

En el discurso pronunciado el 7 de agosto de 2014, en el acto de posesión como presidente de la República para su segundo mandato, el doctor Juan Manuel Santos invita a los colombianos a aprovechar la situación que vive el país, producto de las acciones que se han venido realizando desde su gobierno: «ile llegó la hora a Colombia! Ha llegado la hora de re-imaginar el contrato social que hemos heredado, y las instituciones y políticas que nos han regido. Es nuestra hora... iy vamos a aprovecharla!» (Santos J. M., 2014).

Para reforzar su invitación, acude a un argumento que indica la importancia de su propuesta «Nos cansamos de 'pensar en pequeño'; de creer que estamos condenados a la violencia, a la desigualdad, al atraso, a la falta de oportunidades. iPorque $\mathrm{NO}$ es así!» El motivo del optimismo del presidencial tiene como base la paz, la equidad y la educación, con lo cual considera posible repensar la nación y hacer realizables los sueños.

Esta visión -Colombia en Paz, con Equidad y Educada- es sencilla de recordar, ardua de lograr y profunda en su significado. Y porque depende uno del otro y se refuerzan entre sí. Una paz total no es posible si no hay equidad. Y la única forma de lograr equidad a largo plazo es tener una población bien educada. Además, un país educado es menos propenso a la violencia (Santos J. M., 2014).

Sin embargo, ese sueño requiere de un «nuevo pacto social», el cual pretende que sea a través de una opción distinta e incluyente «Colombia necesita un nuevo pacto social que tome lo mejor de lo que hemos conocido como la izquierda o la derecha para construir -en una Tercera Vía- un país próspero y con equidad» (Santos J. M., 2014). La vía que propone el mandatario colombiano se sitúa en el 
medio de los dos extremos políticos, cuyos radicalismos son considerados como los causantes del conflicto armado interno.

La propuesta, en palabras del presidente, está dirigida a

Un país que cree en la propiedad privada y la protege; un país que fomenta la inversión y el crecimiento económico... pero que lo hace con corazón y con sentido social, para que los más vulnerables, los más pobres, superen su situación y no se queden rezagados (Santos J. M., 2014).

En forma general el mandatario reelecto señala los puntos de su programa de gobierno, que coinciden con lo expuesto en la alocución de su primer acto de posesión como presidente en 2010 y por ende, retoman las estrategias y políticas del denominado Plan Colombia, por cuanto están presentes aspectos relacionados con la economía y el crecimiento de la misma, los aspectos asistenciales para la población vulnerable y la seguridad que, como se ha señalado, son parte de las estrategias del Plan Colombia (Rodriguez V, 2005).

De otra parte, la aplicación de este propósito conlleva a un compromiso: «Que caminemos juntos, que trabajemos juntos, por lo que nunca nos habíamos imaginado como país» (Santos J. M., 2014). En este sentido, el presidente pretende plasmar su programa de gobierno que ha denominado de la Prosperidad democrática y conducir al país a alcanzar la meta de «un país en Paz total... con equidad...y el más educado de América latina» la cual ha de lograrse en una década.

$\mathrm{Al}$ resalta que la paz es el primer pilar de su propuesta política, retoma la frase pronunciada al iniciar su primer mandato «Hace 4 años dije que la puerta del diálogo no estaba cerrada con llave» (Santos J. M., 2014) y pasa a explicar cuál es el contenido de los tres puntos concertados con la guerrilla en el Acuerdo para la Terminación del Conflicto Armado: desarrollo agrario integral, participación política y solución al problema de las drogas ilícitas; además anuncia que se avanza sobre un cuarto aspecto que incluye las víctimas. 
La intervención presidencial deja ver la presencia del Plan Colombia en su programa de gobierno, al señalar que la política de paz estará integrada por la reforma política, la negociación con los grupos armados, las inversiones y acciones para la paz (Rodríguez $\mathrm{V}, 2005)$; aspectos que en líneas generales han comenzado a ser incluidos por el gobierno en las conversaciones con las FARC.

En el punto del Acuerdo sobre las víctimas, el presidente deja sentada su posición frente a la garantía de derechos, al señalar que se han incluido aspectos como la verdad, la reparación y justicia «el paso crucial es poner fin al conflicto para garantizar que no haya más víctimas y que sus derechos puedan ser satisfechos de la mejor manera». Al referirse a la disposición que debe tener la guerrilla, enfatiza... «A diferencia de lo ocurrido en el pasado, en este proceso tiene que haber reparación y se debe esclarecer lo sucedido» (Santos J. M., 2014), lo cual constituye una alusión directa a su antecesor, el expresidente Álvaro Uribe Vélez y al proceso de paz adelantado con los grupos paramilitares.

Como un hecho más del distanciamiento con su antecesor, estable la diferencia entre fin del conflicto y paz «una cosa es poner fin al conflicto y otra es la construcción de la paz, que es lo que tenemos que hacer en las regiones de Colombia» A continuación, señala los compromisos de las FARC y el Gobierno, al respecto dice

Y la paz exige que todos pongan algo de su parte. Las guerrillas tendrán que comenzar -sin rodeos- su proceso de dejación de armas y reincorporación a la vida civil. Y el Gobierno tendrá que poner en marcha todos los mecanismos de acompañamiento y verificación que se acuerden, incluyendo la verificación internacional (Santos J. M., 2014).

De otra parte, el presidente cuestiona a las FARC por actos delictivos atribuidos a esa guerrilla, mientras se adelantan los diálogos:

¿Quién entiende que en La Habana estemos acordando la construcción de acueductos veredales, mientras en Colombia las FARC destruyen esos mismos acueductos - como ocurrió en Granada- o afectan gravemente sus fuen- 
tes de agua -como ocurrió en el Putumayo-? Nadie. Y mucho menos se entiende que sigan causando víctimas civiles, incluyendo niños, como ocurrió en Miranda (Santos J. M., 2014).

Debido a los hechos referidos, advierte la continuidad de la guerra hasta tanto no haya acuerdo, sustenta su determinación en el apoyo de los colombianos y la comunidad internacional

La paciencia de los colombianos y de la comunidad internacional, no es infinita. Señores de las FARC: iestán advertidos! La guerra sigue mientras no se llegue a un acuerdo -eso lo sabemos-, pero... isaquen a los niños, saquen a las mujeres, saquen a los civiles de sus acciones violentas! iNo dinamiten las vías de los campesinos! iNo más pueblos sin luz o sin agua! iNo más petróleo contaminando nuestros ríos!

La advertencia concluye con una exigencia «iActos de paz!... iEso es lo que Colombia pide hoy!» (Santos J. M., 2014).

En el mismo sentido, y como parte de su llamado a la guerrilla, reitera la continuación de sus políticas económicas, sociales y de combate al narcotráfico

Colombia seguirá avanzando en una agenda de justicia social y de construcción de paz; seguirá avanzando en el desarrollo rural, en el fortalecimiento de la democracia, en la lucha contra el narcotráfico, en la reparación de las víctimas... icon o sin las FARC! (Santos J. M., 2014).

Para demostrar su propósito de continuar con sus políticas de gobierno incluidas en la Prosperidad Democrática, especialmente en la reducción de la pobreza, la vivienda gratis, los servicios de salud, la educación, la ampliación de servicios públicos, y vías para el sector rural como forma de facilitar el transporte de productos; remata su disertación recordando la promesa efectuada al comienzo de su primer período de gobierno: «Hace cuatro años dije que a los pobres no les fallaría. iY no les fallamos!» (Santos J. M., 2014).

Con base en la necesidad de continuar con la estrategia de seguridad, justifica el fortalecimiento y modernización de la fuerza pública $\ll$ Y por eso seguiremos fortaleciendo y modernizando a nuestra 
fuerza pública. Ellos han sido guardianes de nuestra democracia y nuestra libertad, y serán -Dios mediante- iguardianes de la PAZ!» (Santos J. M., 2014). Como se ha venido señalando, son permanentes las alusiones al Plan Colombia, toda vez que se conserva la estrategia «para la defensa nacional para reestructurar y modernizar las fuerzas armadas y la policía, para que estos recuperen el estado de derecho, y proporcionen seguridad en todo el territorio nacional» (Rodríguez V, 2005).

$\mathrm{Al}$ término de su intervención, el presidente hace un llamado a «no tenerle miedo al cambio» y por el contrario, soñar y unirse; crear el «futuro que merecemos», el cual es construcción de todos y fruto de los tres ejes de su propuesta de gobierno: Paz, Equidad y Educación.

\section{Puntos de encuentro de los discursos de posesión del Presidente Santos.}

El Presidente Santos en los discursos de toma posesión como mandatario de los colombianos, expresa los lineamientos de la Doctrina de la Seguridad Nacional, en su versión para América latina el Plan Colombia, el cual, como lo señala el investigador Daniel García (1988), desde finales de los ochenta e inicios de los noventa del siglo $\mathrm{XX}$, incluye un nuevo ingrediente para justificar la intervención militar, el apoyo logístico, o la entrega de material bélico y la presencia de asesores norteamericanos en distintos países del continente. El narcotráfico es, sin duda, la nueva amenaza a enfrentar por parte de los Estados Unidos y sus aliados; bajo el pretexto de combatir el tráfico de drogas, se brinda el apoyo para enfrentar los movimientos populares (García, 1988). La forma legal de esta intervención extranjera la constituyó el Plan Colombia; sin embargo, las intervenciones en materia de asesoría militar y apoyo antisubversivo, se venían efectuando desde antes de firmado este pacto entre los Estados Unidos y Colombia. 
Sumado a lo anterior, los dos discursos del presidente Santos, motivo de análisis en este artículo de reflexión, presentan referentes comunes que demuestran el pensamiento ideológico del mandatario en materia de seguridad y cumplimiento de acuerdos internacionales, evidenciando la continuidad de la política de Seguridad Democrática trazada por su inmediato antecesor, la cual está contenida en su propuesta, Prosperidad Democrática, como programa de gobierno.

Los dos programas del presidente Santos en sus dos periodos de gobierno, al estar soportados en la Doctrina de la Seguridad Nacional, incluyen las diez estrategias del Plan Colombia que se sintetizan en:

1. Una estrategia Económica para generar empleo, que fortalezca la capacidad del estado para recaudar impuestos, y que ofrezca una fuerza económica viable para contrarrestar el narcotráfico.

2. Una estrategia fiscal y financiera que adopte medidas severas de austeridad y ajuste con el fin de fomentar la actividad económica, y de recuperar el prestigio tradicional de Colombia en los mercados financieros internacionales.

3. Una estrategia de paz que se apunte a unos acuerdos de paz negociados con la guerrilla con base en la integridad territorial, la democracia y los derechos humanos, que además deban fortalecer el estado de derecho y la lucha contra el narcotráfico.

4. Una estrategia para la defensa nacional para reestructurar y modernizar las fuerzas armadas y la policía, para que estos recuperen el estado de derecho, y proporcionen seguridad en todo el territorio nacional. 
5. Una estrategia judicial y de derechos humanos, con el fin de reafirmar el estado de derecho y para asegurar una justicia igualitaria e imparcial para todos.

6. Una estrategia antinarcóticos, en asocio con los demás países involucrados en algunos o todos los eslabones de la cadena: la producción, distribución, comercialización, consumo, lavado de activos, de precursores y otros insumos, y el tráfico que alimenta la violencia hacia la guerrilla y otras organizaciones armadas.

7. Una estrategia de desarrollo alternativo, que fomente esquemas agropecuarios y otras actividades económicas rentables para los campesinos y sus familias.

8. Una estrategia de participación social que apunte a una concientización colectiva. Esta estrategia busca desarrollar una mayor responsabilidad dentro del gobierno local, el compromiso de la comunidad en los esfuerzos anticorrupción y una presión constante sobre la guerrilla y sobre los demás grupos armados, con el fin de eliminar los secuestros, la violencia y el desplazamiento interno de individuos y comunidades.

9. Una estrategia de desarrollo humano que garantice servicios de salud y educación adecuados para todos los grupos vulnerables de nuestra sociedad durante los próximos años.

10. Una estrategia de orientación internacional que confirme los principios de corresponsabilidad, acción integrada y tratamiento equilibrado para el problema de la droga. Así mismo, el costo de dicha acción y de sus soluciones debe recaer sobre los países involucrados habida cuenta de su capacidad económica individual (Rodríguez V, 2005). 
De otra parte, la propuesta de paz permite evidenciar, como lo señala la investigadora Carolina Rodríguez (2010), la forma como se deja de lado el conflicto, y se crea una identidad entre paz, prosperidad y seguridad

Desconociendo la evidente crisis humanitaria por la que atraviesa el país, portavoces del Gobierno han decretado la no existencia del conflicto. El argumento para hacerlo tiene una inspiración hobbesiana e identifica la paz con la prosperidad y la seguridad con la defensa de la propiedad, la riqueza y la expansión económica...Así, se espera que el discurso sobre la paz tenga efectos inmediatos en el comportamiento de los indicadores económicos (Rodríguez, 2010).

En los discursos del presidente Santos se identifica un concepto de paz como ausencia de guerra, o como no-guerra (Rodríguez, 2010), por lo cual enfatiza en los programas asistenciales, que unidos a las reiteradas advertencias sobre la continuidad de la guerra, el reforzamiento del aparato militar y las conversaciones con las FARC en medio de la confrontación bélica, demuestran su concepción de paz, a la vez, se evidencia la estrategia que apunta a unos acuerdos de paz negociados con la guerrilla con base en la integridad territorial, la democracia y los derechos humanos, que además deban fortalecer el estado de derecho y la lucha contra el narcotráfico, como lo recalca el Plan Colombia. (Rodríguez V, 2005).

\section{Conclusiones}

El Análisis Crítico del Discurso ACD adoptado como metodología para este artículo de reflexión, ha permitido concluir que el uso de recursos y estrategias discursivas del presidente Santos, obedece a la necesidad de enfatizar en su propuesta de terminación del conflicto para persuadir y sensibilizar a la guerrilla acerca de la validez de sus planteamientos y propuestas. A la vez que cumple con los lineamientos del Plan Colombia a través de los programas sociales y 
económicos, incluidos en el programa de gobierno de la Prosperidad Democrática.

La propuesta de paz, expresada en las intervenciones del presidente Santos, devela la existencia de una estrategia tendiente a la derrota militar de la guerrilla, o por lo menos a adelantar las acciones de guerra que conduzcan a su aislamiento, desprestigio y rendición. La paz es entonces una opción que no descarta la guerra, y el diálogo una forma de conservar el modelo económico y social del país, lo cual significa el cumplimiento de las estrategias del Plan Colombia.

El presidente Santos, hace uso de una estrategia de legitimación del poder desde la expectativa de la consolidación de la paz, incluida en su propuesta de gobierno de la Prosperidad Democrática y su llamado a la «Unidad Nacional» como forma de hacer realidad las metas sociales, económicas y consolidar un acuerdo para poner fin al conflicto armado con la guerrilla de las FARC, fortaleciendo el sistema democrático imperante en Colombia.

\section{Bibliografía}

Constitución Política de Colombia. (2016). Bogotá: Legis editores.

Bidegain De Urán, A. M. (1983). Nacionalismo, militarismo y dominación en América. Bogotá: Universidad de los Andes.

Briones, Á. (1978). Economía política del fascismo dependiente. México: Siglo XXI.

Colombia, A. d. (07 de agosto de 2014). Presidencia.gov.co. Recuperado el 16 de enero de 2017, de http://wsp.presidenciagov.co/ Prensa/2010/Agosto/Paginas/20100807_15. aspx

Colombia, C. C. (18 de 05 de 1992). Corte Constitucional. Recuperado el 24 de 02 de 2016, de http://www.corteconstitucional.gov.co/relatoria/1994/T-573-94.htm

Colombia, C. C. (27 de 10 de 1994). Corte Constitucional. Recuperado el 24 de 02 de 2016, de http://www.corteconstitucional.gov.co/relatoria/1994/T-573-94.htm 
Colombia, C. C. (28 de 08 de 2002). Corte Constitucional. Recuperado el 24 de 02 de 2016, de http://www.corteconstitucional.gov.co/relatoria/2002/C-876-02.htm

Colombia, C. C. (28 de 08 de 2002). La Corte Relatoría. Recuperado el 24 de 2 de 2016, de La Corte Relatoría: http://www.corteconstitucional.gov.co/relatoria/2002/C-695-02.htm

Comisión Interamericana, d. (1976). Recuperado el 24 de 03 de 2017, de http://www.oas. org/es/cidh/mandato/documentos-basicos/introducción-documentos-basicos.pdf

Corte Constitucional, C. (28 de 08 de 2002). Corte Constitucional. Recuperado el 24 de 02 de 2016, de http://www.corteconstitucional.gov.co/relatoria/2002/C-695-02.htm

Del Toro, M. (2012). La declaración de derechos humanos: un texto multidimensional. Colección del sistema universal de los derechos humanos., 58-96.

Escobar, L. y. (2011). El Derecho a la paz. ¿Una norma programática, con tendencia a lo normativo 0 a lo semántico? Universitas, 141- 168.

Fairclough, N. y. (2000). Análisis crítico del discurso: una nueva perspectiva. León: Universidad de León.

Fowler, R., Kress, G., \& Hodge, R. y. (1999). De la Lingüística crítica a análisis crítico del discurso: ¿hacia una visión social del lenguaje? Buenos Aires: Universidad de Buenos Aires.

García, D. (1988). Estados Unidos y América latina desde los setentas. Revista Foro No. 7.

Iñiguez, L. (2003). Análisis del discurso. Manual para las ciencias sociales. México: UNAM.

Medrano B, J. L. (04 de 06 de 2012). umanizales.edu.co. Recuperado el 16 de 10 de 2017, de htpp://ridum.umanizales.edu.co:8080/xmlui/bitstream/handle/6789/726/ Jose_Luis_Medrano_\%208_Ago

Naciones, U. (1978). Recuperado el 23 de 12 de 2016, de htpp://www.un.org/es/comun/ docs/?symbol=A/RES/33/73\&Lang $=S$

Naciones, U. (2006). uniandes.edu.co. Recuperado el 30 de 11 de 2015, de https.// cd3.uniandes.educ.co/.../Microsoff\%20Eord\%20-\%20Carta\%20de\%20las\%20N

Rodríguez V, J. (2005). Plan Colombia: Origen, Impacto e Implicaciones. Caracas: Universidad Simón Bolívar.

Rodríguez, C. (2010). ¿Conflicto armado en Colombia? Más allá de la guerra de palabras. Magistro. Revista de Maestría en Educación., 111-125. 
Sánchez, R. (2016). Seis tesis sobre el derecho a la paz en Colombia. Cultura Latinoamericana., 131- 153.

Santos, J. M. (07 de 08 de 2010). Presidencia de la República. Recuperado el 26 de 03 de 2016, de http://wsp.presidencia.gov.co/Discursos/2010/Paginas/Marzo.aspx

Santos, J. M. (07 de 08 de 2014). Presidencia de la República. Recuperado el 26 de 03 de 2016, de http://wsp.presidencia.gov.co/Discursos/2014/Paginas/Agosto.aspx

Stecher, A. (2010). El análisis crítico del discurso como herramienta de investigación psicosocial del mundo del trabajo. Discusiones desde América Latina. Universitas Psychologica.

Tusón, C. \&. (1999). Manual de análisis del discurso. Barcelona: Ariel.

Unesco. (s.f.). portal.unesco.org. Recuperado el 16 de 01 de 2017, de portal.unesco.org: http://portal,uniseco.org/es/ev.php-url_ID =13178\&URL_DO=D0_TOPIC\&URL_ DECTION=201.html

Van Dijk, T. (2003). La multidisciplinariedad del análisis crítico del discurso: un alegato a favor de la diversidad. Barcelona: Gedisa.

Velásquez, E. (2002). Historia de la Doctrina de la Seguridad Nacional. Revista de Ciencias Sociales Universidad Autónoma del Estado de México. México, 11-39.

Wodak, R. (2009). Métodos de análisis crítico del discurso. Barcelona: Gedisa. 